\title{
A New Optical Coherence Tomography Method for Subfoveal Choroidal Thickness Measurement: A Single- center Observational Study
}

\section{Kyung Tae Kang}

Keimyung University Dongsan Medical Center

Ji Hye Jang

Keimyung University Dongsan Medical Center

Yu Cheol Kim ( $\nabla$ eyedr@dsmc.or.kr)

Department of Ophthalmology Keimyung University School of Medicine, Dongsan Medical Center \#1035, Dalgubeoldaero, Dalseo-gu, Daegu 42601, Republic of Korea

\section{Research article}

Keywords: choroid, fovea, optical coherence tomography, retina

Posted Date: October 8th, 2020

DOI: https://doi.org/10.21203/rs.3.rs-87168/v1

License: (c) This work is licensed under a Creative Commons Attribution 4.0 International License. Read Full License

Version of Record: A version of this preprint was published at Medicine on June 18th, 2021. See the published version at https://doi.org/10.1097/MD.0000000000026355. 


\section{Abstract}

Background: Several macular pathologies are associated with choroidal vascular supply, and thus require choroidal thickness evaluation. However, there is no standard subfoveal choroidal thickness (SFCT) measuring method. This study investigated a new method to measure the SFCT, using optical coherence tomography.

Methods: This is a single-center observational study. Eighty-two senior medical students participated as observers and independently measured the SFCT on five standard optical coherence tomography images of healthy adults in a tertiary care setting. They used three different methods: (1) the observer's own method, (2) the conventional method, perpendicular to the retinal pigment epithelium, and (3) the new method, along a virtual line connecting the umbo with the most elevated point of the ellipsoid. Additionally, the SFCT angle-the angle between the measurement line and the vertical line of the image-was measured and compared between methods. The intraclass correlation coefficient was used to determine interpersonal variability.

Results: The intraclass correlation coefficients for SFCT measured by methods 1,2 , and 3 were $0.853,0.880$, and 0.896 , respectively $(P<0.001$ for all). It was the highest with method 3; the intraclass correlation coefficients SFCT angles were $0.647,0.842$, and 0.307 , respectively $(P<0.001$ for all).

Conclusions: The new method showed the lowest interpersonal variability, and could therefore be a reliable standard for SFCT measurement, even in foveae with a steep slope on optical coherence tomography.

Trial registration: Not applicable

\section{Background}

The choroid is a vascular layer between the retina and sclera. It provides blood supply to most ocular tissues, including the outer retinal structures [1]. Choroidal abnormalities, including its loss, thinning, and hyperpermeability, are implicated in various retinal and macular pathologies, such as central serous chorioretinopathy, Vogt-Koyanagi-Harada disease, neovascular age-related macular degeneration, polypoidal choroidal vasculopathy, and diabetic retinopathy [2-6]. Choroidal thickness is a commonly studied parameter [7], along with other choroidal biomarkers such as choroidal volume, choroidal vessel layer thickness, and the choroidal vascularity index. Choroidal thickness is conventionally measured on optical coherence tomography (OCT) from the hyperreflective posterior edge of the retinal pigment epithelium (RPE) to the choroidoscleral interface where the reflectivity changes [8].

Subfoveal choroidal thickness (SFCT), most frequently used to calculate choroidal thickness, can vary widely from 191.5 to $354 \mu \mathrm{m}$ [8-11]. Such variations could be attributed to the software programs used in the OCT systems to map the choroidal thicknesses [8] and a lack of standardization in the measurement techniques. For example, Chhablani et al. [12] measured the choroidal thickness perpendicularly to the RPE layer, while lkuno et al. [9] calculated the choroidal thickness using the vertical distance starting from the RPE layer on the OCT images. These limitations preclude the ability to define the normal cutoff value in subjects without any ocular abnormalities. Hence, there is a need for a standardized choroidal thickness measurement technique to establish the baseline, allow comparisons, and enhance the understanding of disease mechanisms.

\section{Methods}

This study aimed to evaluate a new method to measure SFCT, using enhanced depth imaging (EDI)-OCT, and compare the interpersonal variability among different methods. 
Senior medical students $(n=82)$ at the Keimyung University Medical School volunteered for this non-interventional study during their ophthalmology rotation between January and August 2019. During their attendance, the students were introduced to ophthalmic examination devices, including the OCT system. They reviewed the OCT images of normal subjects and those with various ophthalmic pathologies with the attending professors (KTK and JHJ). Following the introduction and OCT, the students were asked to measure the SFCT on OCT images of five pre-selected standard subjects, using the three methods described below. We have obtained written informed consent from all students before starting the measurements.

\section{Standard Subjects}

For the standard images, five eyes of five pre-selected healthy participants with no proven ophthalmic or systemic ailments were examined with a swept-source OCT (SS-OCT; DRI TRITON, Topcon Corp., Tokyo, Japan). All participants underwent a complete ocular examination, including refraction, slit-lamp biomicroscopy, intraocular pressure, and fundoscopy, to exclude ophthalmic abnormalities. The participants included a 60-year-old man with a spherical equivalent (SE) of +1 diopter as image 1 (Figure 1a), a 67-year-old man with a SE of +2 diopter as image 2 (Figure 1b), a 46-year-old man with a SE of -3.25 diopter as image 3 (Figure 1c), a 43-year-old woman with a SE of -0.375 diopter as image 4 (Figure 1d), and a 58-year-old man with a SE of -11 diopter as image 5 (Figure 1e). These images were chosen as standards because the OCT quality was better than 86/100 as defined by the OCT software. We obtained informed consent from all participants. The institutional review board of Keimyung University approved the study procedures (IRB: 2019-12-056), which adhered to the tenets of the Declaration of Helsinki.

\section{SS-OCT and Scan Protocols}

After pupillary dilation, 64 to 128 averaged horizontal scan images in a 12-mm transverse scan range were obtained with the SS-OCT system for each standard image. The single images were averaged by the system software to improve the image quality. The wavelength-swept laser light source was centered at $1050 \mathrm{~nm}$. The transverse resolution of the OCT was $20 \mu \mathrm{m}$, the in-depth, in-tissue resolution was $8 \mu \mathrm{m}$, and the maximum scan velocity was 100,000 A-scans per second.

\section{Choroidal Thickness Measurement}

SFCT was defined as the distance between the outer border of the hyperreflective line representing the RPE and the choroidoscleral interface. The automated built-in calibration software of the IMAGEnet ${ }^{\circledR} 6$ viewer (Topcon., Tokyo, Japan) was used to determine the distance between these lines. The students were asked to measure the SFCT with the caliper tool embedded in the viewer program. Measurements were performed in three different ways. In method 1, to see an intuitive way of measurement, students that were not yet exposed to the other known method were asked to measure the SFCT, using their own methods, without receiving any specific instructions from the authors (Figure 2a). For method 2, the conventional method, commonly used in previous studies, was followed. The students were asked to measure the SFCT perpendicular to the RPE (Figure 2b). Finally, the new method, or method 3, measured SFCT along a virtual line connecting the deepest point of the fovea (the umbo) and the most elevated point of the ellipsoid zone (the elevation) (Figure 2c). The students were provided a separate room and enough time to perform the SFCT measurements. For interimage and inter-method comparisons, the angle between the SFCT measurement line and the vertical line on the OCT image was calculated using ImageJ ver. 1.50i (National Institutes of Health, Bethesda, MD, USA) (Figure 3).

\section{Interpersonal Variability and Statistical Analyses}

We obtained SFCTs, measured by the 82 independent observers, using three methods in five standard OCT images. The interpersonal variability among the three SFCT measurement methods was assessed using the intraclass correlation coefficient (ICC). Statistical analysis was performed using the Statistical Package for the Social Sciences version 19.0.1 (IBM Corp., Armonk, NY, USA.). Statistical significance was defined as $P<0.05$. 


\section{Results}

The ICC for the SFCT measurements, using the three methods, was 0.853 for method 1, 0.880 for method 2, and 0.896 for method 3 ( $P<0.001$ for all). The interpersonal variability for the SFCT measurements was the lowest with method 3 (Table 1), indicating a high similarity (ICC) between the SFCT values in this group. The angle between the vertical line on the OCT images and the measurement line, termed the SFCT angle, was also compared within each method. The ICC for the SFCT angle was 0.647 for method $1,0.842$ for method 2 , and 0.307 for method $3(P<0.001$ for all). Method 2 had the highest similarity in the ICC for the SFCT angle (Table 1).

The standard deviation of the SFCT was compared between the methods for each standard OCT image. The standard deviation was the smallest in all standard images when method 3 was used, while the results of method 1 and 2 varied between standard images (Table 2).

\section{Discussion}

Noninvasive imaging is widely used to evaluate inaccessible choroidal structures as the RPE light-scattering tendency limits clear choroid visualization [13]. Recent advances in imaging technology, such as EDI-OCT, allow in vivo assessment of the choroidal layers $[8,12,13]$. EDI, introduced by Spaide et al., is obtained by shifting the zero-delay line toward the choroid, leading to improved image resolution and better delineation of the choroidal structures [13]. Many studies analyzing the choroidal thickness used manual measurements that lack standardization [7-9, 13]. Moreover, manual segmentation is time-consuming and is prone to intra- and interobserver variations. Automated segmentation with various machine learning and gradient-based probabilistic methods were developed to overcome this limitation [14-16]. However, these attempts do not directly show the structural dissimilarity between the choroid and the sclera [7]. Therefore, the current study examined a new method to manually measure the SFCT with lower interobserver variability than the conventional method.

The authors suggest measuring the SFCT along the virtual line connecting the foveal umbo and the elevation as it represents the central anatomical point of the fovea. In this study, the measurement of the SFCT along this virtual line had the least interpersonal variability among the three evaluated methods. The new method presented the least interobserver variability regardless of the standard image used, as indicated by its standard deviation, which was the smallest for all images. Methods 1 and 2, on the other hand, presented inconsistent results when assessing the different standard images, as manifested by larger standard deviations.

The mean SFCT in standard images 1, 2, and 3 presented no evident differences between the three measuring methods, but differences were apparent when images 4 and 5 were measured. The SFCT was shorter when measured by method 3. The fovea had a steep slope in these images, while it was gradual in images 1,2 , and 3 . When the fovea has a steep slope, SFCT measurement perpendicular to the RPE can be difficult and might result in overestimating the distance because the slope makes the measurement line longer than the actual distance. Since method 3 presented the least interobserver variability, even in the images with a steep slope, it can be safely assumed to be more reliable than the other two methods.

Another interesting observation was the high interobserver variability in the SFCT angle when measurements were done by the new method. We suspected that the high variability was because the definition of "the deepest point of the fovea" and "the most elevated point of the ellipsoid zone" can be ambiguous to the observers. Method 2 had the most consistent results regarding the SFCT angle because it had a defined angle for SFCT measurement on the images. The SFCT was measured after the observers were introduced to the OCT scans and the structure of the eye as reconstructed on the OCT images. When measuring the SFCT by their own methods (method 1), the observers had no information regarding the conventional measuring method. About one-third of them intuitively measured the SFCT perpendicular to 
the RPE. This could explain why the angle measured by method 1 was less variable than that measured by method 3 . However, since the SFCT measurement by method 3 showed the least variability, the influence of the angle on the actual SFCT measurement could be considered insignificant.

The mean choroidal thickness in this study was auto-segmented and averaged by the OCT system's viewer program in a 1-mm circular area around the foveal center [8]. The authors, however, noticed that the automatic segmentation program failed to delineate the choroidoscleral border between the inner and outer boundaries and to present a valid value for standard images 4 and 5, so the process could not be completed automatically. Until advanced algorithms could accurately and reproducibly measure the SFCT, the authors think that manual SFCT segmentation is required when analyzing OCT images and it has been stated in the previous researches [14-16].

This study has several limitations, as follows: (1) small number of standard images; (2) lack of standard images with chorioretinal pathology; (3) sequential analysis from method 1 to method 3 , which could influence the results because the observers became increasingly familiar with the viewer program; and (4) although eyes with pathology might not have a conspicuous foveal deepest point and/or the most elevated point of the ellipsoid zone, we believe the new SFCT measuring method could guide the development of better measurement methods.

Further studies are warranted to assess the new method's interobserver variability in patients with various chorioretinal pathologies. Regardless, the strengths of the present study include (1) the large number and relative uniformity of the observers, (2) the presence of standard images, and (3) the measurement independence among observers. We considered senior medical students to be well educated to understand the structure of the eye and its appearance on the OCT images to provide reliable SFCT measurements, yet unfamiliar, professionally, with the conventional methods to bias the results. They worked independently to prevent mutual influence during the measurement.

\section{Conclusions}

Measuring the SFCT by the proposed novel method, along the line connecting the deepest foveal point (the umbo) and the most elevated point of the ellipsoid zone (elevation), show lower interpersonal variability than the conventional method, even when the fovea has a steep slope on the OCT image, as is the case in myopic eyes. Therefore the proposed novel method can be a better way of measuring SFCT more reliably than the conventional method.

\section{Abbreviations}

EDI

Enhanced depth imaging; ICC:Intraclass correlation coefficient; OCT:|Optical coherence tomography; RPE:Retinal pigment epithelium; SD:Standard deviation; SE:Spherical equivalent; SFCT:Subfoveal choroidal thickness; SS-OCT:Swept-source OCT.

\section{Declarations}

Ethical approval and consent to participate: The institutional review board of Keimyung University approved this study. All study procedures adhered to the tenets of the Declaration of Helsinki. Informed consent was obtained from all study participants and observers.

Consent for publication: Written informed consent for publication of clinical details and/or clinical images was obtained from the participants. 
Availability of data and materials: The dataset used and/or analyzed during the current study are available from the corresponding author on reasonable request. There are no confidential data or any restrictions on accessing to the original data.

Competing interests: KTK has no competing interests. JHJ reports personal fees from Bayer and Novartis unrelated to the submitted work. YCK reports personal fees from Bayer, Novartis, and Allergan, all unrelated to the submitted work.

Funding: This work was supported by the National Research Foundation of Korea (NRF), the Korean Ministry of Science, ICT, and Future Planning (MSIP) (No. 2014R1A5A2010008). The sponsor had no role in the design or conduct of this research.

Acknowledgments: The authors would like to express their special thanks of gratitude to the senior medical students of the Keimyung University Medical School who volunteered to participate in this study.

Authors' contributions: KTK acquired and analyzed the data and drafted the manuscript. JHJ acquired the data and revised the manuscript. YCK conceived, designed, and supervised the study, and revised the manuscript.

\section{References}

1. Linsenmeier RA, Padnick-Silver L. Metabolic dependence of photoreceptors on the choroid in the normal and detached retina. Invest Ophthalmol Vis Sci. 2000;41:3117-23.

2. Ross A, Ross AH, Mohamed Q. Review and update of central serous chorioretinopathy. Curr Opin Ophthalmol. 2011;22:166-73.

3. Read RW, Rao NA, Cunningham ET. Vogt-Koyanagi-Harada disease. Curr Opin Ophthalmol. 2000;11:437-42.

4. Chung SE, Kang SW, Lee JH, Kim YT. Choroidal thickness in polypoidal choroidal vasculopathy and exudative agerelated macular degeneration. Ophthalmology. 2011;118:840-5.

5. Yannuzzi LA, Sorenson J, Spaide RF, Lipson B. Idiopathic polypoidal choroidal vasculopathy (IPCV). Retina. 2012;32:1-8.

6. Adhi M, Brewer E, Waheed NK, Duker JS. Analysis of morphological features and vascular layers of choroid in diabetic retinopathy using spectral-domain optical coherence tomography. JAMA Ophthalmol. 2013;131:1267-74.

7. Singh SR, Vupparaboina KK, Goud A, Dansingani KK, Chhablani J. Choroidal imaging biomarkers. Surv Ophthalmol. 2019;64:312-33.

8. Hirata M, Tsujikawa A, Matsumoto A, Hangai M, Ooto S, Yamashiro K, et al. Macular choroidal thickness and volume in normal subjects measured by swept-source optical coherence tomography. Invest Ophthalmol Vis Sci. 2011;52:4971-8.

9. Ikuno Y, Kawaguchi K, Nouchi T, Yasuno Y. Choroidal thickness in healthy Japanese subjects. Invest Ophthalmol Vis Sci. 2010;51:2173-6.

10. Manjunath V, Taha M, Fujimoto JG, Duker JS. Choroidal thickness in normal eyes measured using Cirrus HD optical coherence tomography. Am J Ophthalmol. 2010;150:325-9.E1.

11. Margolis R, Spaide RF. A pilot study of enhanced depth imaging optical coherence tomography of the choroid in normal eyes. Am J Ophthalmol. 2009;147:811-5.

12. Chhablani J, Srinivasa PR, Venkata A, Rao HL, Rao BSK, Kumar U, et al. Choroidal thickness profile in healthy Indian subjects. Indian J Ophthalmol. 2014;62:1060-3.

13. Spaide RF, Koizumi H, Pozonni MC. Enhanced depth imaging spectral-domain optical coherence tomography. Am J Ophthalmol. 2008;146:496-500. 
14. Danesh H, Kafieh R, Rabbani H, Hajizadeh F. Segmentation of choroidal boundary in enhanced depth imaging OCTs using a multiresolution texture based modeling in graph cuts. Comput Math Methods Meds. 2014;2014:479268.

15. Alonso-Caneiro D, Read SA, Collins MJ. Automatic segmentation of choroidal thickness in optical coherence tomography. Biomed Opt Express. 2013;4:2795-812.

16. Kajić V, Esmaeelpour M, Považay B, Marshall D, Rosin PR, Drexler W. Automated choroidal segmentation of $1060 \mathrm{~nm}$ OCT in healthy and pathologic eyes using a statistical model. Biomed Opt Express. 2012;3:86-103.

\section{Tables}

Table1

Intraclass Correlation Coefficient of Subfoveal Choroidal Thickness Measured by 82 Observers using Optical Coherence Tomography

\begin{tabular}{|c|c|c|c|c|c|c|}
\hline & \multicolumn{2}{|c|}{ Method $1^{a}$} & \multicolumn{2}{|c|}{ Method $2^{b}$} & \multicolumn{2}{|c|}{ Method $3^{c}$} \\
\hline & ICC & $\begin{array}{l}P \text { - } \\
\text { value }\end{array}$ & ICC & $\begin{array}{l}P \text { - } \\
\text { value }\end{array}$ & ICC & $\begin{array}{l}P \text { - } \\
\text { value }\end{array}$ \\
\hline Subfoveal choroidal thickness $(\mu \mathrm{m})$ & 0.853 & $<.001$ & 0.880 & $<.001$ & 0.896 & $\dot{0} 001$ \\
\hline $\begin{array}{l}\text { Angle between the vertical line and measurement line } \\
\text { (degrees) }\end{array}$ & 0.647 & $\begin{array}{l}<.001 \\
0.01\end{array}$ & 0.842 & $\dot{0} 001$ & 0.307 & $\begin{array}{l}< \\
0.001\end{array}$ \\
\hline
\end{tabular}

ICC, intraclass correlation coefficient.

a Observers' own methods; ${ }^{b}$ Perpendicular to the retinal pigment epithelium; ${ }^{\mathrm{C}}$ The virtual line connecting the deepest point of the fovea and the most elevated point of the ellipsoid zone.

Table 2

Method-based Mean SFCT Measured from Optical Coherence Tomography by 82 Observers

\begin{tabular}{|c|c|c|c|c|c|c|c|c|c|c|}
\hline \multirow{2}{*}{$\begin{array}{l}\text { SFCT } \\
\text { measurement } \\
\text { method }\end{array}$} & \multicolumn{2}{|c|}{ Image 1} & \multicolumn{2}{|c|}{ Image 2} & \multicolumn{2}{|c|}{ Image 3} & \multicolumn{2}{|c|}{ Image 4} & \multicolumn{2}{|c|}{ Image 5} \\
\hline & Mean & SD & Mean & SD & Mean & SD & Mean & SD & Mean & SD \\
\hline Method $1^{a}$ & 349.25 & 27.812 & 273.86 & 32.324 & 253.78 & 21.432 & 290.53 & 32.026 & 121.11 & 54.049 \\
\hline Method $2^{b}$ & 351.73 & 27.637 & 278.14 & 26.092 & 250.77 & 30.217 & 299.00 & 27.622 & 124.44 & 42.759 \\
\hline Method $3^{c}$ & 339.44 & 25.144 & 258.30 & 24.071 & 253.52 & 20.605 & 268.52 & 25.635 & 104.16 & 42.271 \\
\hline
\end{tabular}

SD, standard deviation; SFCT, subfoveal choroidal thickness

a Observers' own methods; ${ }^{b}$ Perpendicular to the retinal pigment epithelium; ${ }^{c}$ The virtual line connecting the deepest point of the fovea and the most elevated point of the ellipsoid zone.

\section{Figures}



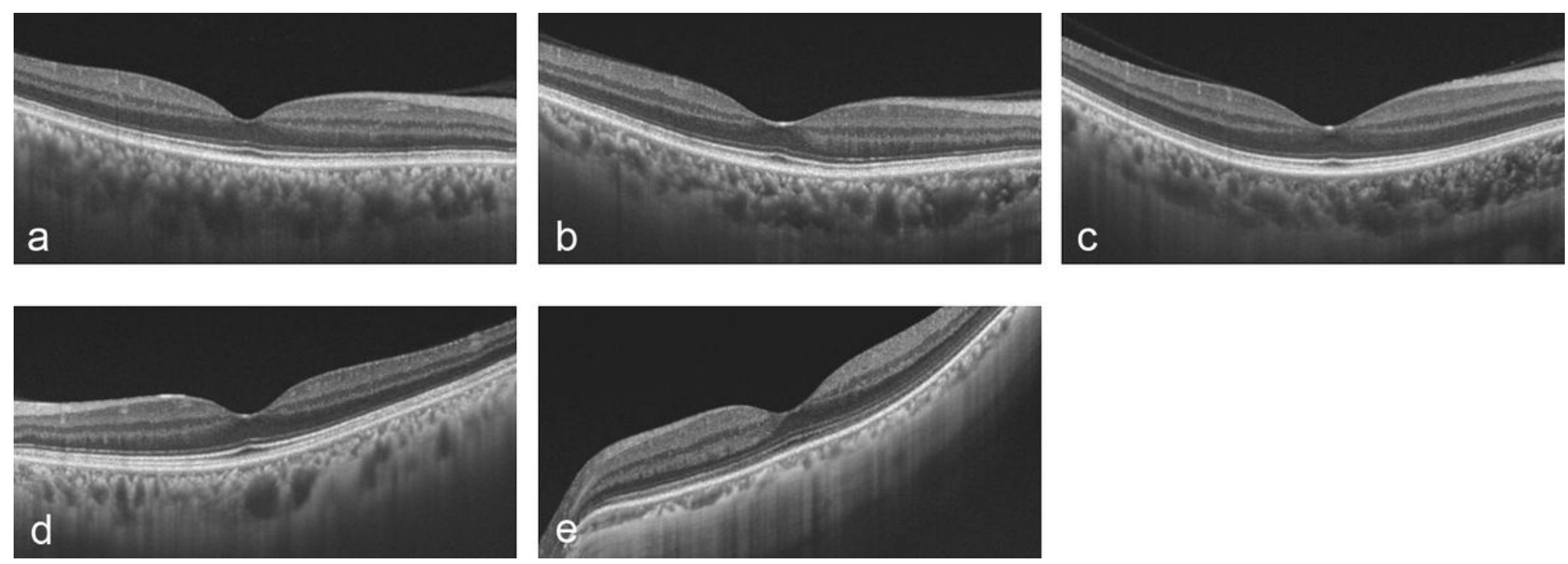

\section{Figure 1}

Standard multi-averaged horizontal swept-source optical coherence tomography images. The 12-mm transverse scan range images of five pre-selected standard healthy subjects are presented (a-e). Each image was obtained by averaging 64 to 128 single optical coherence tomography images to improve the quality.
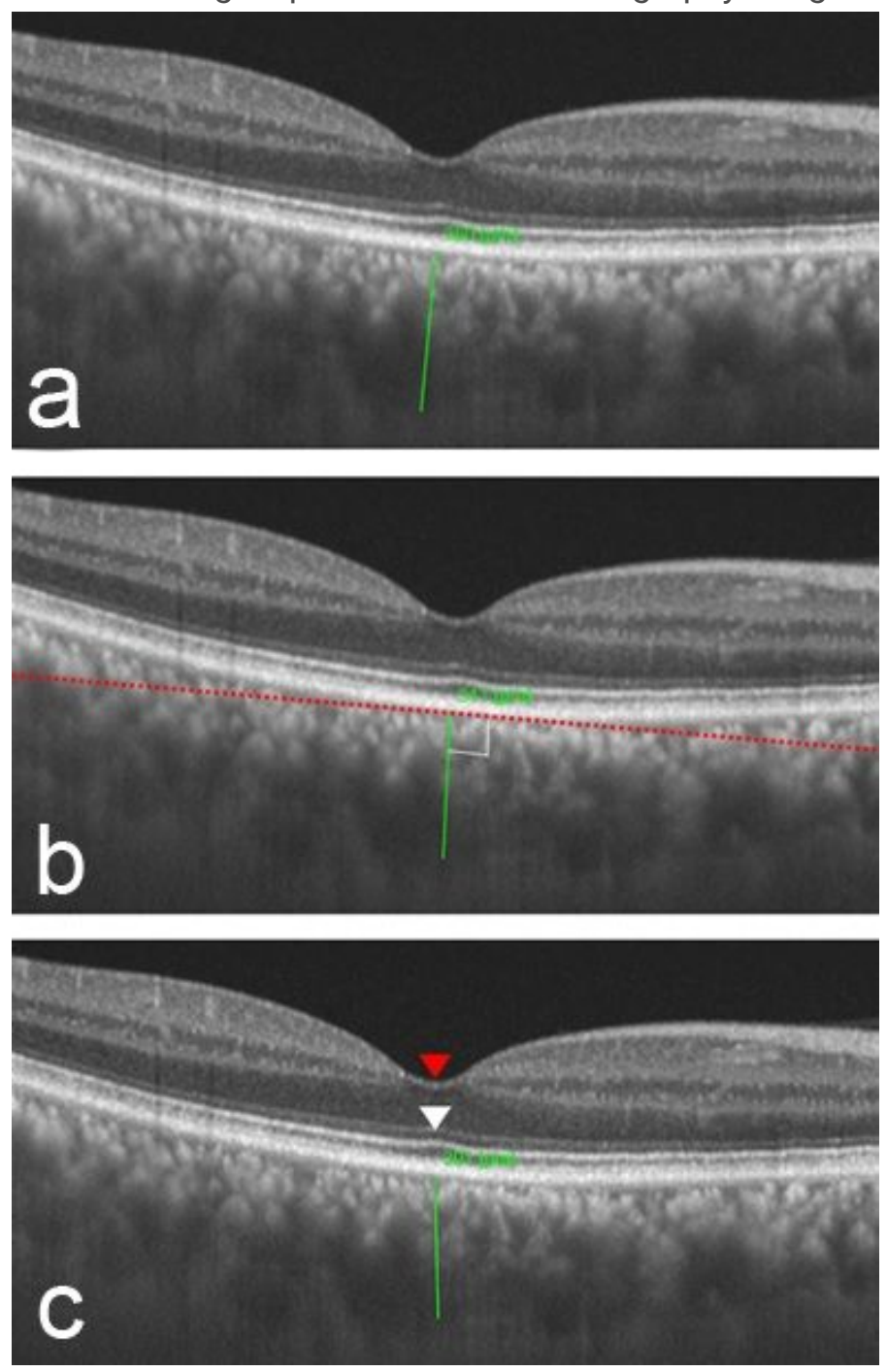

Figure 2 
Three methods of subfoveal choroidal thickness measurement using a representative image. a. Method 1, the measurement (green line) between retinal pigment epithelium and the choroidoscleral interface was done by the observer's own method. b. Method 2, the measurement (green line) was done perpendicular to the retinal pigment epithelium (red dots). c. Method 3, the measurement (green line) was done along a virtual line connecting the deepest point of the fovea (the umbo, red arrowhead) and the most elevated point of the ellipsoid zone (the elevation, white arrowhead).

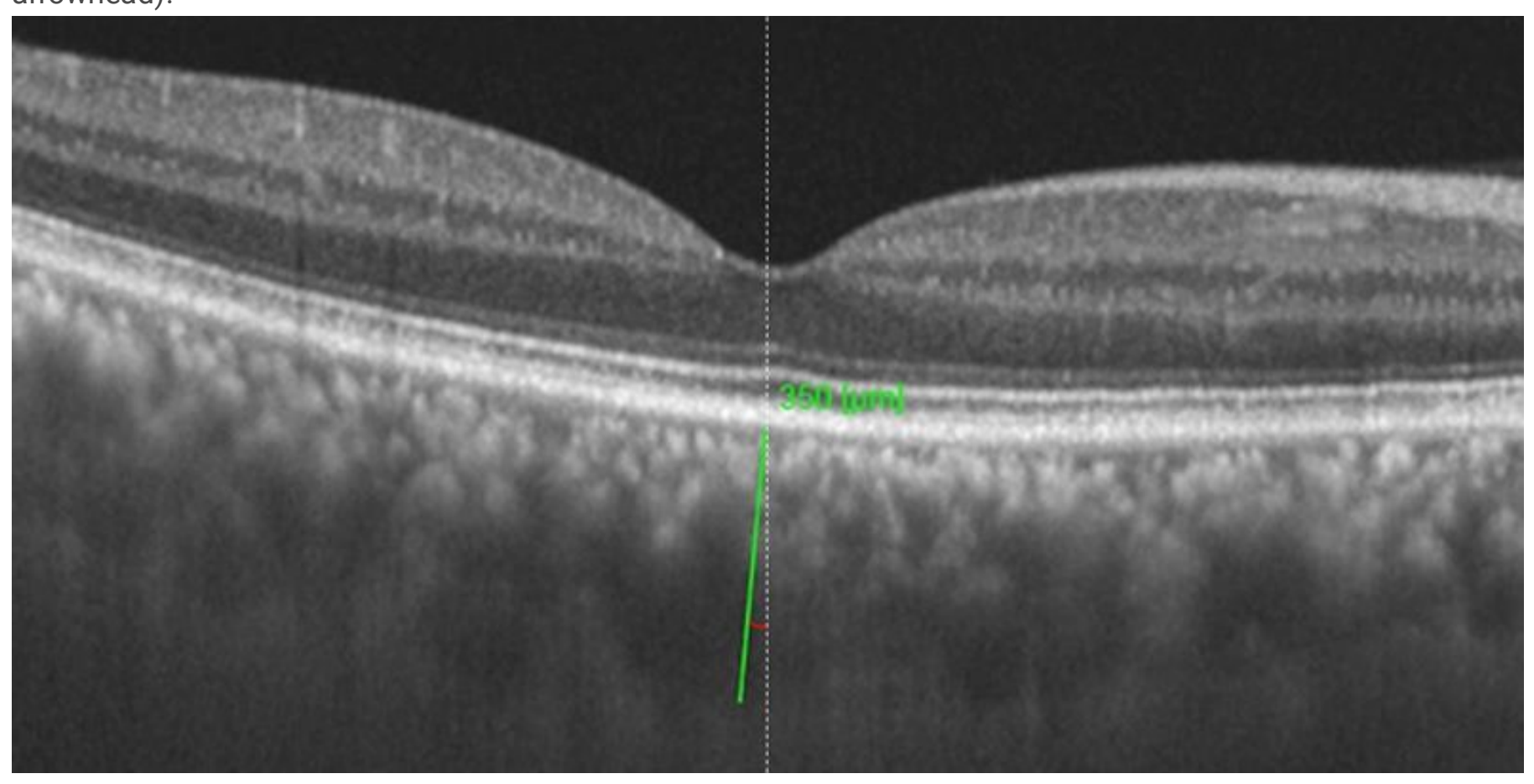

\section{Figure 3}

Angle measurement: measurement of the angle (red marking) between the vertical line (white dashed line) on the image and the subfoveal choroidal thickness measurement line (green line). 